Tropical Journal of Pharmaceutical Research April 2012; 11 (2): 209-215

(C) Pharmacotherapy Group,

Faculty of Pharmacy, University of Benin Benin City, 300001 Nigeria.

All rights reserved.

Available online at http://www.tjpr.org

Research Article

http://dx.doi.org/10.4314/tjpr.v11i2.6

\title{
Anti-Fatigue Effects of Methazolamide in High- Altitude Hypoxic Mice
}

\author{
Gang Zhang $^{1-3 \star}$, Si-Min Zhou ${ }^{1-3}$, Jun-Huai $\operatorname{Tian}^{1-3}$, Qing-Yuan Huang ${ }^{2-4}$ and \\ Yu-Qi Gao-4 \\ ${ }^{1}$ Department of High Altitude Military Hygiene, College of High Altitude Military Medicine, Third Military Medical \\ University, ${ }^{2}$ Key Laboratory of High Altitude Medicine, Ministry of Education, ${ }^{3}$ The Key Laboratory of High Altitude \\ Physiology and High Altitude Disease, PLA, ${ }^{4}$ Department of Pathophysiology and High Altitude Physiology, College \\ of High Altitude Military Medicine, Third Military Medical University, Chongqing 400038, China.
}

\begin{abstract}
Purpose: To investigate the anti-fatigue property of methazolamide (MTZ) in high-altitude anoxic mice. Methods: Mice fatigued by high-altitude hypoxia were housed in a hypobaric chamber (equivalent to a low pressure chamber of $5000 \mathrm{~m}$ altitude) for 10 consecutive days. The anti-fatigue property of MTZ was evaluated by exhaustive swimming test, determination of blood concentration of lactic acid and sugar as well as blood urea nitrogen (BUN), and liver glycogen.

Results: Our findings indicate that the administration of MTZ can prolong swimming capacity time and improve exercise tolerance as well as increase the content of liver glycogen, reduce the level of lactic acid in muscles, when compared with anoxic control group. MTZ also delayed the accumulation of BUN, compared with anoxic control.

Conclusion: The results show that MTZ has anti-fatigue effects in mice, and further suggest that it is a potential novel remedy for fatigue due to high-altitude hypoxia.
\end{abstract}

Keywords: Methazolamide; Anti-fatigue; Exhaustive exercise; High-altitude hypoxia. 


\section{INTRODUCTION}

Fatigue may be defined as a situation in which the capacity for work is diminished and efficiency of accomplishment reduced. It is usually accompanied by a feeling of physical or mental tiredness, resulting from severe stress and hard physical or mental work [1,2]. It is a common symptom in people with stressful contemporary lifestyle [3,4]. High altitude environments could have adverse effects such as high-altitude cerebral edema and high-altitude pulmonary edema and fatigue $[5,6]$ on the normal functioning of people accustomed to living at low altitudes.

Fatigue can be more serious due to hypoxia, impair daily functioning and lead to negative effects on quality of life. Patients need to take treatments to deal with fatigue, hasten recovery from fatigue, and prevent long-term and chronic fatigue. Alleviation of fatigue by self-medication is one of the most common and effective ways.

With the increasing development of western China, more and more people live in the high altitude environment of that region. Therefore, alleviation of fatigue caused by high-altitude hypoxia has become a serious problem that requires urgent attention. In the past few decades, health scholars and athletic physiologist have sought nutritional materials and tonic drugs, including natural active products and synthetic chemicals, to improve athletic ability, postpone fatigue and accelerate the elimination of fatigue in human beings [7-9]. However, drugs to counter fatigue are in short supply because the effects of present drugs are not very acceptable or resources are limited.

Methazolamide (MTZ) is a carbonic anhydrase inhibitor which has been used in oral formulations to treat glaucoma (primary open-angle glaucoma, angle-closure glaucoma and secondary glaucoma, among others) due to their ability to lower intraocular pressure by reducing aqueous humour formation $[10,11]$. To the best of our knowledge, there have been no reports on the anti-fatigue effects of MTZ. In this study we have evaluated the anti-fatigue activity of MTZ in mice.

\section{EXPERIMENTAL}

\section{Materials}

MTZ tablets were obtained from Hang Zhou Aoyipollen Pharmaceutical Co, Ltd (Hang Zhou, China). Glycogen assay kit and blood urea nitrogen assay kit were purchased from Sigma (St Louis, USA). Blood sugar meter and portable blood lactate analyzer (Lactate Pro ${ }^{T M}$ LT-1710) were purchased from Arkray (Tokyo, Japan) and Roche (Basel, Switzerland), respectively.

\section{Experimental animals}

Experimental groups consisted of ten ICR mice (weighing $20 \pm 2 \mathrm{~g}$ ) per group. The mice were randomly divided into six groups - a normoxic control group, an anoxic group and four treatment groups. The mice in the treatment groups were gavaged with 4 doses of $0.312,0.937,4.687$ and $9.375 \mathrm{mg} / \mathrm{kg}$, respectively, this being $0.3,1.0,5.0$ and 10.0 times of the normal dose of MTZ (0.937 $\mathrm{mg} / \mathrm{kg}$ ), respectively, in humans. The mice in the normoxic control group were housed under controlled conditions of $25 \pm 1{ }^{\circ} \mathrm{C}$ and $60 \%$ relative humidity. The mice in the rest groups were housed in a hypobaric chamber (equivalent to a low pressure chamber of $5000 \mathrm{~m}$ altitude). The experimental protocols were approved by the Animal Care and Use Committee of our institute. The animals were handled according to standard protocols for the use of laboratory animals [12]. After administration for 10 consecutive days, biochemical parameters were evaluated.

\section{Exhaustive swimming test}

One hour after the last oral administration, exhaustive swimming test (EST) was carried out as previously described $[13,14]$, with some modifications. Briefly, the mice were dropped individually into an acrylic plastic pool $(90 \times 60 \times 60 \mathrm{~cm})$ filled with fresh 
water, maintained at $25 \pm 1{ }^{\circ} \mathrm{C}$, approximately $50 \mathrm{~cm}$ deep so that mice could not support themselves by touching the bottom with their tails. A lead block (5\% of body weight) was attached to the tail root of each mouse. The swimming to exhaustion time was used as the index of exhaustive swimming capacity. The mice were assessed to be exhausted when they failed to rise to the surface of water to breathe within a 10 -s period.

\section{Determination of blood sugar and lactic acid}

One hour after the last oral administration, blood lactic acid (BLA) and blood sugar (BS) concentrations were determined at 3 time points, namely, before swimming, after exhaustion and $20 \mathrm{~min}$ after exhaustion. Blood samples were collected from mouse tail tip and a portable blood lactate analyzer (Lactate Pro ${ }^{\text {TM }}$ LT-1710, ARKRAY) was used to determine BLA. Blood lactate area under curve (BL-AUC) was calculated using Eq 1.

$$
B L-A U C=5 \times(A+3 \times B+2 \times C) \ldots .(1)
$$

where $\mathbf{A}=\mathrm{BLA}$ before swimming; $\mathbf{B}=\mathrm{BLA}$ after exhaustion; and $\mathbf{C}=\mathrm{BLA} 20 \mathrm{~min}$ after exhaustion.

A blood glucometer (Roche) was employeded to determine the BS.

\section{Determination of blood urea nitrogen}

One hour after rest from the swimming test, the animals were anesthetized with sodium pentobarbital, and $0.5 \mathrm{ml}$ of blood was collected by extirpating the left eyeball. The blood sample was cooled for about $3.5 \mathrm{~h}$ at $4^{\circ} \mathrm{C}$ and serum obtained prepared by centrifugation at a speed of $2000 \mathrm{rpm} 4^{\circ} \mathrm{C}$ for $15 \mathrm{~min}$. The level of blood urea nitrogen (BUN) was determined by automatic biochemical analyser using commercial kits.

\section{Determination of liver glycogen}

One hour after the last oral administration, the mice were sacrificed. The liver was removed, homogenized and its glycogen (LG) concentration was determined with an automatic biochemical analyser using commercial kits.

\section{Statistical analysis}

The results are expressed as mean \pm S.E.M. Statistical was analysis was by SPSS software (SPSS for Windows 15.0, SPSS Inc, USA). Statistical difference was determined by one-way ANOVA, followed by LSD-t test for multi-group comparisons. Probability values of $p<0.05$ were considered significant.

\section{RESULTS}

\section{Effect of MTZ on swimming-to-exhaustion time}

The results of the effect of MTZ on swimming-to-exhaustion time are shown in Fig 2. The swimming time to exhaustion of $0.3,1.0$ and 5.0 times of the normal dose of MTZ (0.312, 0.937 and $4.687 \mathrm{mg} / \mathrm{kg}$ ) were significantly longer than the anoxic control group $(p<0.01)$, and the normal dose of MTZ $(0.937 \mathrm{mg} / \mathrm{kg})$ had the optimum effect among all the doses, and followed by the mice given 0.3 and 5.0 times of the normal dose of MTZ, 0.312 and $4.687 \mathrm{mg} / \mathrm{kg}$, respectively.

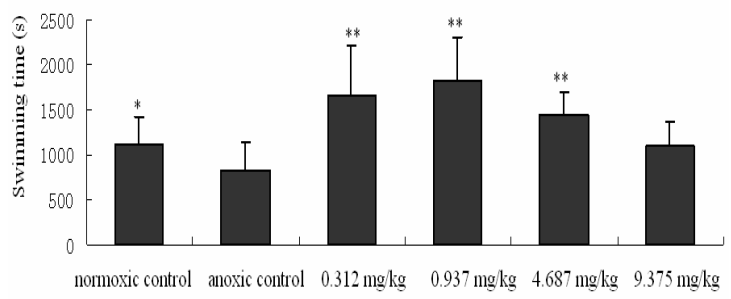

Fig 1: Effect of MTZ on swimming-to-exhaustion time of mice. Experiment was carried out one hour after last oral administration; values are mean \pm S.E.M. $(\mathrm{n}=10) ;{ }^{\star} p<0.05$, and ${ }^{\star *} p<0.01$, compared with anoxic control. 


\section{Effect of MTZ on blood lactic acid and blood sugar levels}

The results of the effect of MTZ on blood lactic acid and blood sugar are shown in Fig 2 and Table 1, respectively. The concentration of blood lactic acid was expressed as blood lactate area under curve (BL-AUC). As can be seen from Fig 2, MTZ possesses significant blood lactate acid lowering potential in high-altitude hypoxic mice model, which was exhibited in a dosedependent manner (0.937 - $9.375 \mathrm{mg} / \mathrm{kg})$.

The data for blood sugar level are presented in Table 1. The effect of MTZ on physical fitness is expressed as the maintenance of blood sugar level. One-way ANOVA indicate that there were no significant differences between the blood sugar levels of the mice in the MTZ groups, compared with anoxic control group.

\section{Effect of MTZ on blood urea nitrogen}

Blood urea nitrogen (BUN) data are presented in Fig 3. The BUN of MTZ treatment groups decreased significantly, compared with normoxic control group, in a dose-dependent manner $(0.937-9.375$ $\mathrm{mg} / \mathrm{kg}$ ).

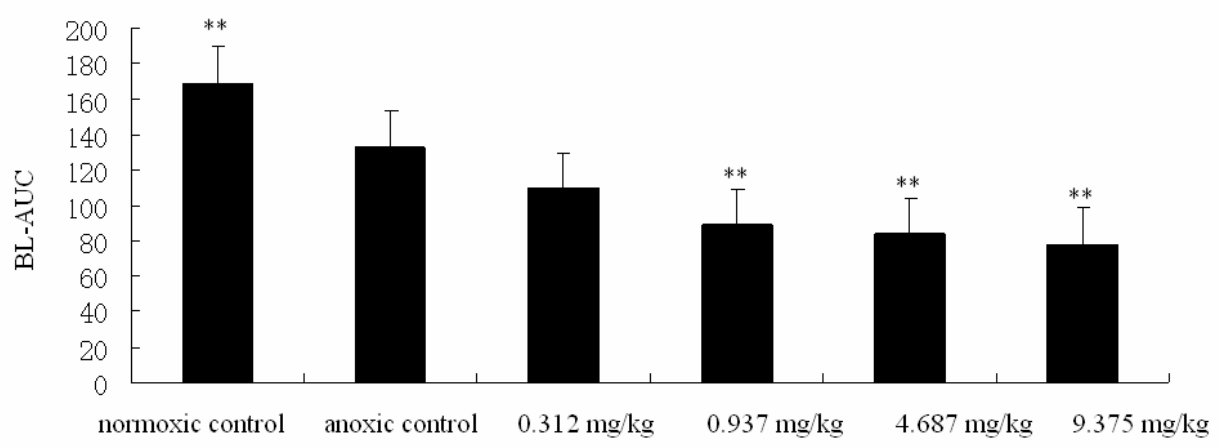

Fig. 2: Effect of MTZ on the blood lactic acid of hypoxic mice.

$B L-A U C=$ blood lactate area under curve. Blood lactic acid concentration (BLA) was determined at 3 time points (before swimming, after exhaustion and 20 min after exhaustion); BL-AUC was derived BLA data; values are mean \pm S.E.M. $(n=10) .{ }^{* *} p<0.01$, compared with anoxic control.

Table 1: Effect of MTZ on blood sugar level of high-altitude hypoxic mice

\begin{tabular}{lccc}
\hline Group dose & \multicolumn{3}{c}{ Concentration of blood sugar (mmol/L) } \\
(mg/kg) & Time $\boldsymbol{A}$ & Time $\boldsymbol{B}$ & Time $\boldsymbol{C}$ \\
\hline Normoxic control group & $6.42 \pm 2.01$ & $5.72 \pm 2.00$ & $5.98 \pm 2.11$ \\
Anoxic control group & $5.22 \pm 1.09$ & $4.45 \pm 1.53$ & $4.54 \pm 1.46$ \\
0.312 & $5.81 \pm 1.51$ & $6.06 \pm 2.25$ & $6.30 \pm 2.85$ \\
0.937 & $5.57 \pm 1.15$ & $4.72 \pm 2.24$ & $4.58 \pm 1.92$ \\
4.687 & $5.92 \pm 1.32$ & $4.89 \pm 1.86$ & $4.80 \pm 2.37$ \\
9.375 & $6.12 \pm 1.82$ & $5.06 \pm 1.59$ & $5.02 \pm 2.11$ \\
\hline
\end{tabular}

Key: Time $A$ = before swimming; Time $B=$ after exhaustion; Time $C=20$ min after exhaustion. The vehicle (control, $10 \mathrm{~mL} / \mathrm{kg}$ ) or MTZ $(0.312,0.937,4.687$ and $9.375 \mathrm{mg} / \mathrm{kg}$ ) was administered orally; values are mean \pm S.E.M. $(n=10)$ 


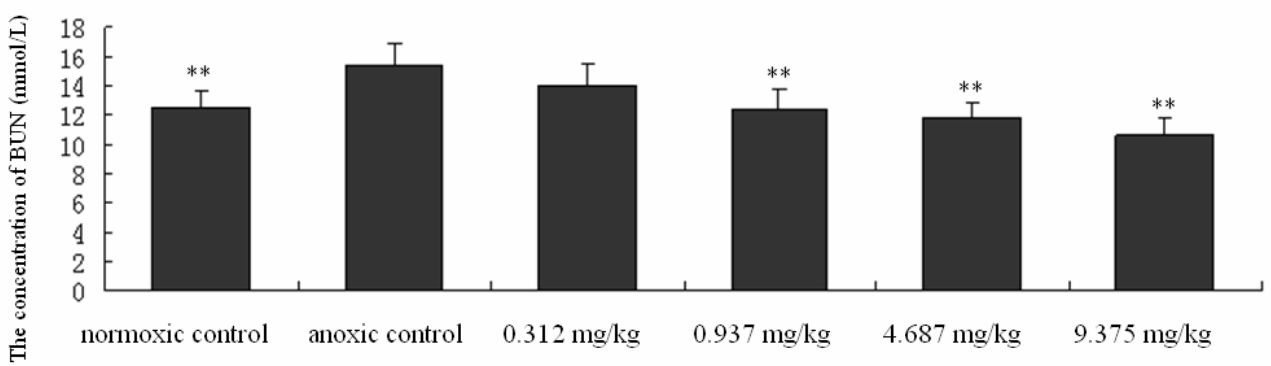

Fig 3: Effect of oral MTZ on blood urea nitrogen (BUN) of high-altitude hypoxic mice. Values are mean \pm S.E.M. $(n=10) ;{ }^{* *} p<0.01$, compared with the anoxic control group.

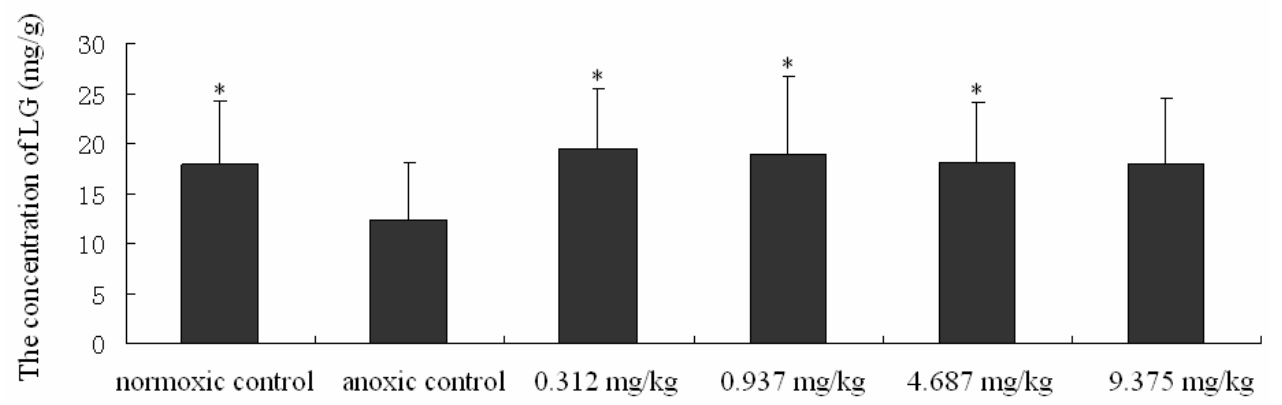

Fig 4: Effects of the MTZ on liver glycogen of high-altitude hypoxic mice. Values are mean \pm S.E.M. $(n=$ $10) ;{ }^{*} p<0.05$, compared with the anoxic control group

\section{Effect of MTZ on liver glycogen}

Liver glycogen content of the mice after MTZ administration is depicted in Fig 4. The liver glycogen content of the mice (given 0.3, 1.0 and 5.0 times of the normal dose in humans) groups was significantly higher than that of the anoxic control group $(p<0.05)$.

\section{DISCUSSION}

In recent years, more people are suffering from fatigue due to high-altitude hypoxia. Fatigue could be induced following forced exercise, sickness, heat stroke or sleep disturbance, and fatigue often results in symptoms related to CNS functions including reduced activity and diminished muscle endurance aswell as impaired brain-related functions such as concentration, attention and memory $[15,16]$. Drugs can produce antifatigue effects by supplying energy substances, accelerating the elimination of metabolic products, adjusting the internal environment, enhancing body immunity or directly stimulating the nervous system [13]. However, there are very few pharmacological agents or therapies available for the treatment of fatigue [17].

The findings for mice subjected to housing in a hypobaric chamber (equivalent to the low pressure chamber of $5,000 \mathrm{~m}$ altitude) for 10 consecutive days show that swimming capacity time was significantly shortened. The length of time taken to swim to exhaustion indicates the degree of fatigue [18]. MTZ administration prolonged swimming capacity time and improved exercise tolerance, indicating that the fatigue could be relieved by MTZ. It seems that MTZ can be effective when given alone to relieve and treat fatigue.

Trop J Pharm Res, April 2012;11 (2):213 
The anaerobic glycolysis product, lactic acid, is an important biochemical parameter related to fatigue, and the accumulation of blood lactic acid is considered a major inducer of muscle fatigue $[19,20]$. The muscle produces much lactic acid when it obtains enough energy from anaerobic glycolysis, and the increased concentration of lactic acid brings about a reduction of $\mathrm{pH}$ in muscle tissue and blood. In addition, lactic acid is known to induce relaxation of vascular smooth muscle in various organs [21]. Consequently, the redundant lactic acid causes a reduction of $\mathrm{pH}$ in muscle tissue, and also induces many side effects, such as fatigue, which are harmful to body performance. Therefore, reduction in the accumulation of lactic acid in muscle is beneficial for alleviation of fatigue $[7,22]$. In this study, the MTZ significantly lowered blood lactatic acid level in a dose-dependent manner.

Furthermore, MTZ treatment attenuated the increase in blood urea nitrogen (BUN) which is a metabolic product of proteins and amino acids. BUN is a sensitive index for evaluating exercise tolerance and fatigue status. In otherwords, the content of serum BUN of human bodies rises with increase in exercise load $[7,23,24]$. In the present study, administration of MTZ lowered BUN level, compared to anoxic control, thus suggesting that MTZ reduces the catabolism of protein for energy, increases adaptive capacity to exercise load, and ultimately improves endurance capacity.

Energy for exercise is derived initially from the decomposition of glycogen which is the form of carbohydrate stored in the liver [25]. Lactic acid is a product of glycolysis under anaerobic conditions, and the accumulation of lactic acid pre-disposes to fatigue. Thus, liver glycogen is an important biochemical parameter related to fatigue. Hypoxic mice administrated with MTZ had even more liver glycogen stores compared with anoxic control group. This suggests that administation of MTZ increases liver glycogen content post- exercise by improving glycogen reserve or by reducing the consumption of glycogen during exercise, thereby reducing the level of the anaerobic glycolytic product, lactic acid.

\section{CONCLUSION}

The present study demonstrates that administration of MTZ can prolong $t$ swimming capacity time and improve exercise tolerance by increasing liver glycogen content and reducing lactic acid levels in muscle. The drug also delays the accumulation of BUN (the metabolism product of protein and amino acid). Therefore, MTZ has some anti-fatigue effects and may, therefore, be of benefit to people suffering from fatigue caused by high-altitude hypoxia. However, investigation of the parameters related to fatigue at cellular and molecular levels are required. It would also be necessary to elucidate the exact mechanisms by which MTZ exerts its effects.

\section{ACKNOWLEDGEMENT}

This work was supported by the Key Project of the National Research Program of China (no. 2009BAI85B06) and Military Key Scientific and Technological project (no. 08G081).

\section{REFERENCES}

1. Akazawa KH, Cui Y, Tanaka M, Kataoka Y, Yoneda $Y$, Watanabe $Y$. Mapping of regional brain activation in response to fatigue-load and recovery in rats with c-Fos immunohistochemistry. Neurosci Res 2009; 66: 372-379.

2. Chaudhuri $A$, Behan PO. Fatigue in neurological disorders. Lancet 2004; 363: 978-988.

3. Ream E, Richardson A. Fatigue: a concept analysis. Int J Nutr Stud 1996; 33: 519-529.

4. Watanabe $Y$, Evengard $B$, Natelson $B H$, Jason $L A$, Kuratsune $H$ (Eds.), Fatigue Science for Human Health, edn 1. 2008: Springer, Tokyo, pp. $V-X I$.

5. Frisancho AR. Functional adaptation to high altitude hypoxia. Science 1975; 187: 313-319.

6. Hackett $P H$, Roach RC. High-altitude illness. New Engl J Med 2001; 345: 107-114.

7. Huang LZ, Huang BK, Ye Q, Qin LP. Bioactivityguided fractionation for anti-fatigue property of

Trop J Pharm Res, April 2012;11 (2):214 
Acanthopanax senticosus. J Ethnopharmacol 2011; 133: 213-219.

8. Ma L, Cai DL, Li HX, Tong BD, Song $L H$, Wang $Y$. Anti-fatigue effects of salidroside in mice. $J$ med coll PLA 2008; 23: 88-93.

9. Mizuma H, Tanaka M, Nozaki S, Mizuno K, Tahara $T$, Ataka S, Sugino $T$ Shirai $T$, Kajimoto $Y$, Kuratsune $H$, Kajimoto $O$, Watanabe $Y$. Daily oral administration of crocetin attenuates physical fatigue in human subject. Nutr Res 2009; 29: 145-150.

10. Maren TH, Haywood JR, Chapman SK, Zimmerman $T J$. The pharmacology of methazolamide in relation to the treatment of glaucoma. Invest Ophth Vis Sci 1977; 16: 730-742.

11. Fridriksdottir $H$, Loftsson $T$, Stefansson $E$. Formulation and testing of methazolamide cyclodextrin eye drop. J Control Release 1997; 44: 95-99.

12. National Institute of Health, USA. Public health service policy on humane care and use of laboratory animals; 2002.

13. Wang $L$, Zhang $H L$, Lu R, Zhou YJ, Ma R, Lv JQ, Li $X L$, Chen LJ, Yao $Z$. The decapeptide CMS001 enhances swimming endurance in mice. Peptides 2008; 29: 1176-1182.

14. Mizunoya W, Oyaizu S, Ishihara K, Fushiki $T$. Protocol for measuring the endurance capacity of mice in an adjustable-current swimming pool. Biosci Biotechnol Biochem 2002; 66: 1133-1136.

15. Chen JR, Wang TJ, Huang HY, Chen LJ, Huang YS, Wang YJ, Tseng G.F. Fatigue reversibly reduced cortical and hippocampal dendritic spines concurrent with compromise of motor endurance and spatial memory. Neuroscience 2009; 161: 1104-1113.
16. Afari $N$, Buchwald $D$. Chronic fatigue syndrome: a review. Am J Psychiatry 2003; 160: 221-236.

17. Uthayathas $S$, Karuppagounder SS, Tamer SB, Parameshwaran K, Degim T, Suppiramaniam $V$, Dhanasekaran M. Evaluation of neuroprotective and anti-fatigue effects of sildenafil.Life Sci 2007;81: 988-992.

18. Tanaka M, Nakamura F, Mizokawa S, Matsumura A, Nozaki S, Watanabe Y. Establishment and assessment of a rat model of fatigue. Neurosci Lett 2003; 352: 159-162.

19. Edman KA, Lou F. Changes in force and stiffness induced by fatigue and intracellular acidification in frog muscle fibres. J Physiol 1990; 424: 133-149.

20. He LY, Lou MM, Yan WX. The experimental study of antifatigue of health foods. Chin J Food Hyg 1997; 9: 1-6.

21. McKinnon W, Aaronson PI, Knock G, Graves J, Poston L. Mechanism of lactate-induced relaxation of isolated rat mesenteric resistance arteries. J Physiol 1996; 490: 783-792.

22. Tang $W$, Zhang $Y$, Gao J, Ding $X$, Gao $S$. The antifatigue effect of 20(R)-ginsenoside $R g 3$ in mice by intranasally administration, Biol Pharm Bull 2008; 31: 2024-2027.

23. Zhang $Y$, Yao $X$, Bao $B$, Zhang $Y$. Anti-fatigue activity of a triterpenoidrich extract from Chinese bamboo shavings (Caulis bamfusae in taeinam). Phytother Res 2006, 20: 872-876.

24. Ding JF, Li YY, Xu JJ, Su XR, Gao X, Yue FP. Study on effect of jellyfish collagen hydrolysate on anti-fatigue and anti-oxidation. Food Hydrocolloid 2011, 25:1350-1353.

25. Suh SH, Paik IY, Jacobs K. Regulation of blood glucose homeostasis during prolonged. Mol Cells 2007; 23: 272-279. 